Article

\title{
Angular momentum and energy transfer in a Whitehead's theory of gravity
}

\author{
L. Acedo \\ Instituto Universitario de Matemática Multidisciplinar \\ Building 8G, $2^{\circ}$ Floor, Camino de Vera, \\ Universitat Politècnica de València, 46022, Valencia, Spain.
}

\section{Introduction}

Keywords: Energy and angular momentum transfer; Modified theories of gravity; Anomalous increase of the astronomical unit

PACS classifications: $\quad$ 04.80.-y; 04.80.Cc; 04.50.Kd

\begin{abstract}
In this paper, we revisit a modified version of the classical Whitehead's theory of gravity in which all possible bilinear forms are considered to define the corresponding metric. Although, this is a linear theory that fails to give accurate results for the most sophisticated predictions of general relativity, such as gravity waves, it can still provide a convenient framework to analyze some new phenomena in the Solar System. In particular, recent development in the accurate tracking of spacecraft and the ephemerides of planetary positions have revealed certain anomalies in relation with our standard paradigm for celestial mechanics. Among them the so-called flyby anomaly and the anomalous increase of the astronomical unit play a prominent role. In the first case the total energy of the spacecraft changes during the flyby and a secular variation of the semi-major axis of the planetary orbits is found in the second anomaly. For this to happen it seems that a net energy and angular momentum transfer is taken place among the orbiting and the central body. We evaluate the total transfer per revolution for a planet orbiting the Sun in order to predict the astronomical unit anomaly in the context of Whitehead's theory. This could lead to a more deeply founded hypothesis for an extended gravity model.
\end{abstract}


Although the General Theory of Relativity (GR) has been spectacularly confirmed throughout the years thanks to carefully designed experiments and observations [1], there are reasons to believe that GR is not the last word in our understanding of gravity, even from a purely classical macroscopic point of view. In particular, the recent discovery of a set of possible anomalies, still not laid out on firm phenomenological foundation, are opening a new era of high precision measurements and tests on gravitational theory as well as on our understanding of celestial mechanics in the Solar System. For a complete review of these anomalies the reader can check the review by Iorio [2].

In this paper, we will be concerned with two, still unresolved, anomalies in the ephemeris of the Solar System: (i) the so-called anomalous increase of the Astronomical Unit (au) (ii) the anomalous increase of the eccentricity of the Moon's orbit. Krasinsky and Brumberg firstly reported the first one in 2004. By a careful analysis of ephemeris data from different sources in the last decades, including radar ranging obtained with spacecraft, they found an unexplained increase in the value of the fundamental distance scale of the Solar System, the astronomical unit, with a rate of $15 \pm 4$ meters per century [3]. By adding new recent measurements, Standish gave a lower, but still significant, value for the secular increase of the au of $7 \pm 2$ meters per century [4]. The definition of the Astronomical Unit has changed through time. The early approach by Gauss was obtained by writing Kepler's third law in the form:

$$
k^{2}(1+M) T^{2}=4 \pi^{2} a^{3},
$$

where $T$ is the length of the year measured in terms of the mean solar day, $M$ is the mass of the sun in terms of the Earth's mass and $a$ is the semi-major axis of the Earth's orbit in meters. Here, $k$ is the so-called Gaussian gravitational constant. In the first approach to the problem of defining the au it was assumed that $k$ is a constant by definition and from there we can obtain the size of the orbit from Eq. (1) but since 2012 is exactly defined as $149597870.7 \mathrm{kms}$. This was established as a definition in the Resolution B2 of the XXVIII of the general assembly of the International Astronomical Union [5]. It is clear that by a mere redefinition of the au we do not eliminate the problem of its secular increase because it simply manifests in another orbital parameter.

Since the announcement of Krasinsky and Brumberg there have been a series of attempts to explain or make sense of this anomaly in the context of standard physics with the aid of modified gravity models. In 2005, Iorio suggested that the effect could arise in the context of the Dvali-Gabadadze-Porrati multidimensional braneworld scenario [6], an explanation based on the kinematics of Finsler geometry was provided by Li and Chang [7]. A more conventional effect in the form of the implications of cosmological expansion in a McVittie space-time was considered by Arakida [8] but the resulting increase of the semi-major axis of the planets is 9 orders of magnitude smaller than the estimation by Kransinsky and Brumberg or Standish. An alternative explanation based upon the classical angular momentum conservation law was proposed by Miura et al. [9]. According to these authors a tidal mechanism, similar to that involved in the tidal recession of the Moon from the Earth [10], would be responsible for the increase of the planetary axes. Anyway, no physical mechanism is detailed in the paper for the generation of the tides in the solar plasma in order to verify if they could really be sufficiently high to account for the anomalous effect. Another nonconventional approximation to the problem was given by Acedo in terms of a variable speed of light model [11] and by Bel in terms of a time-varying gravitational constant [12]. 
A possibly related problem in Solar system ephemeris was found thanks to the improvement on modern techniques on lunar laser ranging (LLR). This LLR technique has allowed for the monitoring of Moon's orbit with unprecedented accuracy for a period of several decades. In 2009, Williams and Boggs presented their results on the analysis of 38 years of LLR observations from 1970 to 2008 [14,15]. By using the standard models for the Lunar and Earth interiors and all the tidal dissipative processes they were able to explain the observations save for an anomalous increase of the eccentricity of the orbit in the range $(9 \pm 3) \times 10^{-12}$ per year. Further LLR data extending until 2013 was also studied more recently with the improved DE430 ephemerides and the anomaly was still present but with a reduced value of $(5 \pm 2) \times 10^{-12}$ [16]. The authors of these works are confident that improved ephemerides and models of the Solar and Lunar interior and the geological processes would suffice to explain away this lingering anomaly. Nevertheless, there are also reasons to think that non-standard physics or unnoticed subtle effects still unmodelled could be the origin [17,18].

Iorio considered some possible classical phenomena that could be involved in this anomaly. In particular, he showed that the general relativistic gravitomagnetic acceleration has the correct order of magnitude but, on the other hand, its secular effect vanishes so it cannot explain the anomaly. Other alternatives include the perturbations on the Earth-Moon system by a massive trans-Plutonian planet still undiscovered. The problem with this hypothesis is that the mass of this planet should be so large (for example and Earth mass planet at $30 \mathrm{au}$ ) that it would have already been discovered [17]. An interesting unconventional idea is the proposal of Iorio of a universal radial acceleration of the form:

$$
\mathbf{A}=k H_{0} v_{r} \hat{\mathbf{r}}
$$

where $H_{0}=7.47 \times 10^{-11} \mathrm{yr}^{-1}$ is the Hubble constant at the present epoch, $v_{r}=d r / d t$ is the radial velocity of the planet or satellite towards the central body and $k$ is a constant of the order of unity. This perturbation predicts the Astronomical unit increase and the lunar eccentricity anomaly for $2.5 \leq k \leq 5$ [18]. Acedo also suggested that the coincidence of the prefactor in Eq. (2) with the Hubble constant could arise in an extended post-newtonian model of gravity [19].

A strange fact related with the phenomena of the flyby anomalies which has sparked so much interest in recent years $[21-26,43]$ is that it seems to be associated with an anomalous energy transfer among the spacecraft and the Earth. The same could be said of the anomalous increase of the astronomical unit. In classical physics several mechanism are known that can allow for the transfer of energy or momentum from a rotating object to an orbiting satellite or spacecraft. The best studied is the tidal friction caused by the bulge raised by the Moon's gravity on the Earth oceans and crust and its displacement due to Earth's rotation around its axis. This dissipation gives rise to a lag on the bulge with respect to its ideal position on the Earth-Moon axis [27]. Because of this process the Moon recedes, approximately, $3.7 \mathrm{~cm}$ per year from the Earth as has been accurately tested with the LLR technique [14].

Another classical mechanism that has recently been applied to the flyby of a spacecraft is the coupling among the tesseral harmonics of the rotating Earth with the spacecraft along its flyby. The density inhomogeneities along the parallels of the Earth gives rise to a time-dependent potential that causes a net energy transfer with a spacecraft that follows a hyperbolic trajectory [28]. In standard general relativity we have also the Penrose mechanism for a particle crossing the ergosphere of a Kerr black hole [29]. But 
the possible energy/momentum transfer leading to an anomalous increase of the astronomical unit or the flyby anomaly cannot be attributed to any of these phenomena.

In this paper we will revisit an extended model of Whiteheadian gravity in order to elucidate the mechanism for energy and momentum transfer between the orbiting and the central body and its possible consequences for anomalous perturbations in the Solar system.

\section{An extended Whitehead's gravity model}

In the early years of Einstein's development and proposal of the General Theory of Relativity there were other competing formulations for a relativistic theory of gravity. The Finnish theoretical physicist Gunnard Nordström tried to find a consistent framework for a theory of gravity within the context of the Special Theory of Relativity [30]. Einstein himself closely followed some of these hypotheses [31]. Another model for a theory of gravity formulated over the basis of the Special Theory of Relativity was proposed by the philosopher and mathematician A. N. Whitehead in a book originally published in 1922 [32,33]. The idea of Whitehead is that space-time is fundamentally Minkowskian and that gravity emerges from a symmetric covariant tensor, $g_{\mu \nu}, \mu, \nu=0,1,2,3$, which is invariant under the Poincaré group. In contrast with the General Theory of Relativity of Einstein, this tensor has nothing to do with the structure of space-time. The theory of Whitehead has received attention and even praise many years after its formulation [33-35] but it is now clear that it cannot be a serious contender to General Relativity, particularly in what concerns gravity waves [36]. Anyway, as a toy model of gravity it can still provide some insights into local phenomena in the scale of the Solar system.

Whitehead's starting point is a null vector that takes into account the retardation delay from the propagation of a signal from the source to the test point:

$$
L^{\alpha}=x^{\alpha}-\hat{x}^{\alpha},
$$

where $x^{\alpha}$ are the space-time coordinates of the test particle and $\hat{x}^{\alpha}$ are the coordinates of the intersection of the past cone corresponding to the event $x^{\alpha}$ and the world line, $\mathcal{W}$, of the point like source. We now define the fourth-velocity along this world line, $u^{\alpha}$, a scalar $r$ and a reduced null vector $l^{\alpha}$ as follows:

$$
\begin{aligned}
r & =-u_{\alpha} L^{\alpha}, \\
l^{\alpha} & =\frac{1}{r} L^{\alpha} .
\end{aligned}
$$

Whitehead does not develop a field equation for the coupling between matter and spacetime curvature as Einstein did. On the other hand, his theory is much simpler, being totally contained in the definition of a symmetric covariant tensor:

$$
g_{\mu \nu}=\eta_{\mu \nu}-\frac{2 G m}{c^{2} r} l_{\mu} l_{\nu}
$$

where $\eta_{\mu \nu}$ is the diagonal Minkowski's metric: $\eta_{00}=-1, \eta_{11}=\eta_{22}=\eta_{33}=1, \eta_{\mu \nu}=0, \mu \neq \nu$. From this metric many classical predictions of General Relativity can be deduced [33].

In this paper we are not going to use the original theory of Whitehead but a recent extension of it developed by Bel $[37,38]$. By using the notation above and all possible combinations of $l_{\alpha}$ as defined 
in Eq. (4) and the fourth-velocity, $u_{\alpha}$, the following general expression for the symmetric tensor $g_{\mu \nu}$ is found:

$$
g_{\mu \nu}=\eta_{\mu \nu}+\frac{1}{r}\left(A_{0} u_{\mu} u_{\nu}+A_{1} \eta_{\mu \nu}+A_{2}\left(l_{\mu} u_{\nu}+l_{\nu} u_{\mu}\right)-A_{3} l_{\mu} l_{\nu}\right)
$$

where $\eta_{\mu \nu}$ denotes Minkowski's metric as usual and $A_{0}, \ldots, A_{3}$ are a series of constants to be determined. Bel imposed the condition given by Einstein's vacuum equations, $R_{\mu \nu}=0$, to find the relation $A_{0}=2\left(A_{1}-A_{2}\right)$. The Newtonian limit also implies that $A_{1}-A_{3}=2 G m$. Here $G$ is the gravitational constant and $m$ the mass of the source.

By taking the limit in which the spatial components of the velocities are small compared with the speed of light, Bel also found the following equations of motion [38,41]:

$$
\frac{d w^{i}}{d t}=-\frac{G m}{L^{3}} L^{i}+\frac{G m}{c L^{2}}\left(u^{i}-3 \frac{L^{i} u_{i}}{L^{2}} L^{i}\right)=f^{i},
$$

where $L^{2}=L_{i} L^{i}, w_{i}$ are the spatial components of the velocity of the test particle and $u^{i}, i=1,2,3$ are the spatial components of the velocity of the source. Notice that the first term on the right-hand side of Eq. (7) is the standard Newtonian force $-\frac{G m}{L^{3}} L^{i}$. The second term would be the anomalous force predicted by the extended Whitehead's theory.

The objective of this work is to evaluate the forces corresponding to this anomalous component exerted by the orbiting body upon the mass distribution of the central body and the resulting balance of energy and momentum transfer.

\section{Anomalous momentum transfer}

Here we calculate the total system of anomalous forces and the corresponding momentum exerted by an orbiting planet over the whole body of the Sun according to Eq. (7). The geometry of the problem is displayed in Fig. 1. To simplify the calculations we make three reasonable assumptions: (i) The rotation axis of the Sun is at right angles with the orbiting plane of the Earth (the so-called ecliptic plane in astronomy) (ii) The orbit of the Earth is, approximately, circular and the modulus of the velocity is a constant $\mathbf{u}$ (iii) The density of the Sun is constant throughout its volume.

We consider separately the two terms beyond the Newtonian approximation in Eq. (7). The first term is given by $f_{I}^{i}=G M /\left(c L^{2}\right) u^{i}$ so, for the situation in Fig. 1, it would be given by:

$$
\mathbf{f}_{I}=\frac{G M}{c L^{2}} u_{y} \hat{\jmath}
$$

so the contribution of this force (per unit mass) to the total momentum of the system of anomalous forces acting upon the mass elements of the central body (with coordinates $x, y$, and $z$ ) are:

$$
\mathcal{M}_{I}=\mathbf{r} \times \mathbf{f}_{I}=\frac{G M}{c L^{2}} u_{y}(x \hat{k}-z \hat{\imath}),
$$

where the $\mathbf{L}$ vector is related to the position vectors for the Earth and the mass element inside the Sun by: $\mathbf{L}=\mathbf{r}-\mathbf{D}$. So, the modulus satisfies the relation:

$$
L^{2}=r^{2}+D^{2}-2 \mathbf{r} \cdot \mathbf{D}=r^{2}+D^{2}-2 r D \cos \theta
$$


Figure 1. Geometry for the calculation of the anomalous momentum exerted by the Earth upon the Sun. We assume that the rotation axis of the Sun is normal to the ecliptic plane and that the orbit of the Earth is circular and that it moves with linear velocity of constant modulus $|\mathbf{u}|$. We notice that $\mathbf{r}$ denotes the radius vector corresponding to an arbitrary point inside the Sun (denoted by $P$ ), $\mathbf{L}$ is the vector from the center of the Earth to that point and $\mathrm{D}$ is the vector from the Earth to the center of the Sun.

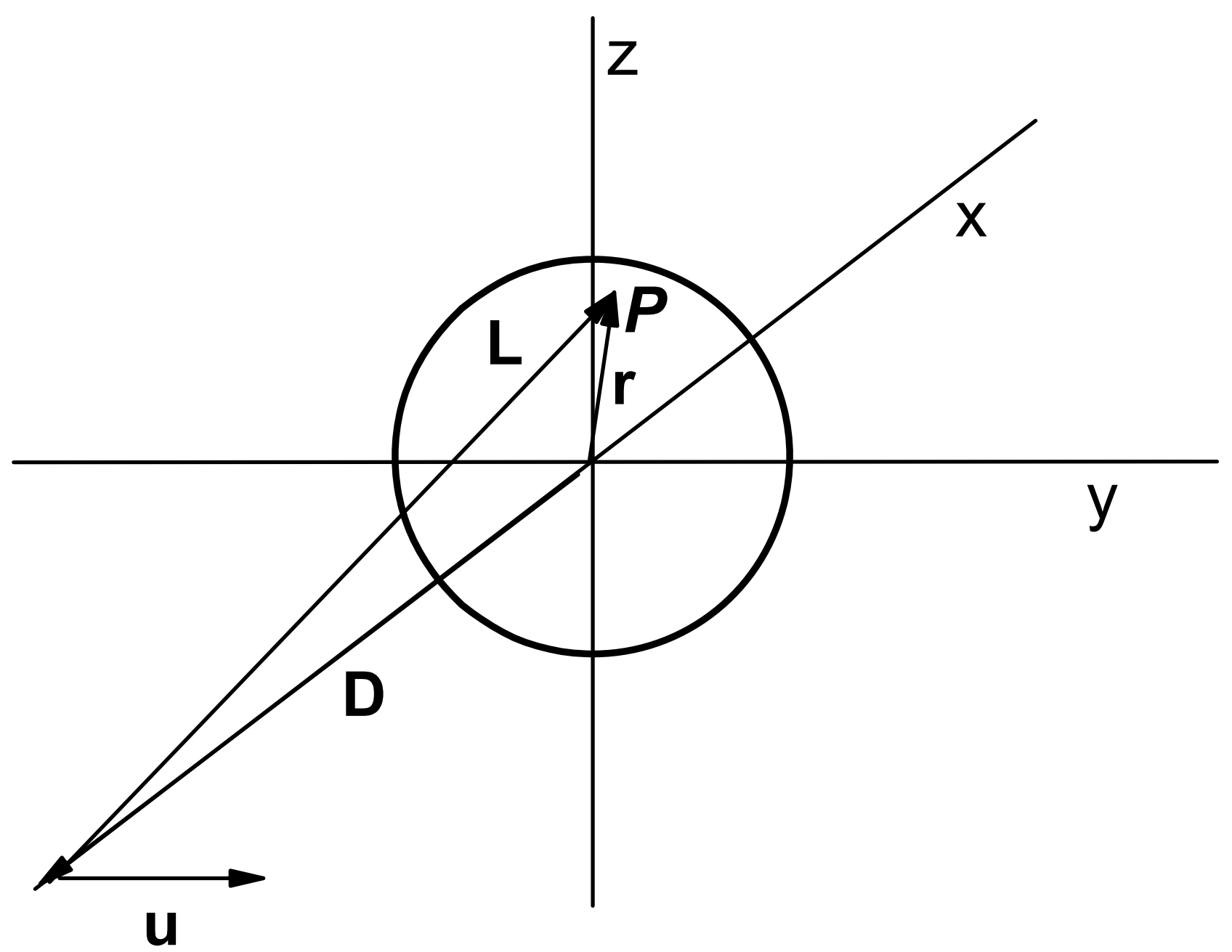


In this relation we have ignored the retardation effects in the definition of the vector $L^{\alpha}$. Notice that here $\theta$ is the polar angle of the point $P$ with respect the axis $x$. Integrating now over the whole volume of the Sun we have:

$$
M_{I}=\int_{\mathcal{V}} \mathcal{M}_{I} \rho d^{3} \mathbf{r}
$$

Moreover, by using assumption (iii) of constant's mass density inside the Sun, we have:

$$
M_{I}=\hat{k} \frac{2 \pi G M}{c} \rho u_{y} \int_{r=0}^{R} d r \int_{\theta=0}^{\pi} d \theta \frac{r^{3} \sin \theta \cos \theta}{r^{2}+D^{2}-2 r D \cos \theta} d \theta .
$$

$R$ being the radius of the Sun. By evaluating the double integral we arrive at the following expression for the first term of the total torque of the anomalous system of forces:

$$
\boldsymbol{M}_{I}=\hat{k} \frac{2 \pi G M}{c} u_{y} D^{2} \rho \mathcal{F}\left(\frac{R}{D}\right)
$$

where

$$
\mathcal{F}(x)=\frac{3 x}{4}-\frac{7 x^{3}}{12}+\frac{1}{8}\left(3-2 x^{2}-x^{4}\right) \ln \left(\frac{1-x}{1+x}\right) .
$$

In Eq. (13) we have ignored the term in the $\hat{\imath}$ direction because its integral is null by symmetry. Similarly, for the second term of the anomalous force in Eq. (7) we can write:

$$
\mathbf{f}_{I I}=-\frac{3 G M}{c L^{4}}(\mathbf{L} \cdot \mathbf{u}) \mathbf{L}=-\frac{3 G M}{c L^{4}} u_{y} r \sin \theta \cos \phi(\mathbf{r}-\mathbf{D}),
$$

where $\theta$ is the polar angle with respect to axis $x$ and $\phi$ is the azimuthal angle measured in the plane $y-z$ with reference at axis $y$. Therefore, the contribution of this force applied at point $P$ to the total momentum of the system is given by:

$$
\mathcal{M}_{I I}=\frac{3 G M u_{y}}{c L^{4}} r \sin \theta \cos \phi \mathbf{r} \times \mathbf{D}=\frac{3 G M u_{y}}{c L^{4}} r \sin \theta \cos \phi(D z \hat{\jmath}-D y \hat{k}) .
$$

Integrating over all the mass elements in the Sun and ignoring the $\hat{\jmath}$ component that cancels out by symmetry, as before, yields:

$$
\boldsymbol{M}_{I I}=-\hat{k} \frac{3 \pi G M D}{c} u_{y} \rho \int_{r=0}^{R} d r r^{4} \int_{\theta=0}^{\pi} d \theta \frac{\sin ^{3} \theta}{\left(r^{2}+D^{2}-2 r D \cos \theta\right)^{2}} .
$$

An explicit expression can also be found for the integral in Eq. (17), yielding:

$$
\begin{aligned}
\mathcal{Q}(x) & =\frac{x}{\left(1-x^{2}\right)^{2}}\left[x\left(1+x^{4}\right)+\frac{\pi}{2} \frac{x^{2}\left(1+x^{2}\right)\left(1+2 x^{2}\right)}{1-x^{2}}-\frac{\pi}{4}\left(1+x^{6}\right) \frac{2 x^{2}}{1-x^{2}}\right] \\
& -\frac{\left(1+x^{2}\right)^{2}\left[\left(1-x^{2}\right)^{2}-2 x^{2}\right]}{2 x^{3}\left(1-x^{2}\right)^{3}} x^{4} \arctan \left(\frac{2 x}{1-x^{2}}\right),
\end{aligned}
$$

and from Eqs. (13), (14), (17) and (18) we arrive at the following equation for the total momentum of the extra forces acting upon the central spherical body of radius $R$ and constant density, $\rho$ :

$$
\boldsymbol{M}=\hat{k} \frac{2 \pi G M}{c} \rho u_{y} D^{2}\left\{\mathcal{F}\left(\frac{R}{D}\right)-\frac{3}{2} \mathcal{Q}\left(\frac{R}{D}\right)\right\}
$$

where we must remember that $M$ corresponds to the mass of the orbiting body but $\rho$ is the density of the central body. 
Table 1. Parameters for the Sun, the Earth and the Moon and their orbits obtained from Ref. [39].

\begin{tabular}{lccc} 
Parameter & Sun & Earth & Moon \\
\hline Gravitational constant $\left(\mathrm{m}^{3} / \mathrm{s}^{2}\right)$ & $1.327124 \times 10^{20}$ & $3.986004 \times 10^{14}$ & $4.90283 \times 10^{12}$ \\
mass $\left(10^{24} \mathrm{~kg}\right)$ & 1988500 & 5.9724 & 0.07346 \\
Density $\left(\mathrm{kg} / \mathrm{m}^{3}\right)$ & 1408 & 5514 & 3344 \\
Moment of inertia $\left(I / M R^{2}\right)$ & 0.070 & 0.3308 & 0.394 \\
Angular rotational velocity $(\mathrm{rad} / \mathrm{s})$ & $2.86533 \times 10^{-6}$ & $7.29211 \times 10^{-5}$ & $2.6617 \times 10^{-6}$ \\
Orbital velocity $(\mathrm{km} / \mathrm{s})$ & n.a. & 29.78 & 1.022 \\
Mean orbital distance $(\mathrm{km})$ & n.a. & 149597870.7 & 378000 \\
\hline
\end{tabular}

4. Application to the Solar system anomalies Here we will apply the results for the anomalous momentum transfer among an orbiting body and the central one to the Earth-Sun and Moon-Earth systems. We will see that the predictions of the force model in Eq. (7) are unrealistically large unless we generalize it further in the form:

$$
\mathbf{f}_{\text {non-Newtonian }}=\beta(D) \frac{G m}{c L^{2}}\left(\mathbf{u}-3 \frac{\mathbf{L} \cdot \mathbf{u}}{L^{2}} \mathbf{L}\right)
$$

where $\beta(D)$ is a scalar parameter with a possible dependence on the average distance between the orbiting and the central body. Some useful parameters to apply the results in Sec. 3 are listed in Table 1.

If we use now the parameters in Table 1 and Eqs. (19), (14), (18) with $R$ being the radius of the Sun, $D$ the orbital distance to the Earth, GM the mass constant of the Earth and $\rho$ the density of the Moon we obtain that the momentum of the system of forces acting upon the Sun is $\boldsymbol{M}_{\text {Sun }}=-2.70036 \times$ $10^{29} \beta(D) \hat{k}$ Joules. As this momentum is opposite to the angular velocity vector of the Sun, it means that it tends to decrease the total angular momentum of the Sun and its rotational energy and, consequently, it corresponds to an increase of the orbital energy of the Earth to keep the energy balance. The decrease of the rotational energy of the Sun is then given by:

$$
\frac{d E_{\mathrm{rot}}}{d t}=\Omega_{\mathrm{Sun}} M_{\mathrm{Sun}}=-7.73741 \times 10^{23} \beta(D) \text { Joules } / \mathrm{s}
$$

The anomalous increase of the astronomical unit can also be correlated with an increase of the orbital energy of the Earth in the form [40]:

$$
\frac{d E_{\text {orb }}}{d t}=-\frac{d a}{d t} \frac{1}{a} E=\frac{d a}{d t} \frac{G M_{\text {Sun }} m_{\text {Earth }}}{2 a^{2}},
$$

and from the revised value of $d a / d t$ given by Standish [4] as an average of 7 meters per century as well as the data in Table 1 we obtain $d E / d t=3.92804 \times 10^{13}$ Joules per second. In order for the energy transfer rates in Eqs. (21) and (22) to coincide we can estimate the dimensionless parameter $\beta$ as $\beta(D)=5.0767 \times 10^{-11}$. We notice also that corrections to the value of $\beta(D)$ can be obtained with 


\subsection{The anomalous increase of the orbital eccentricity In this section we will analyze the case of the} Earth-Moon system in order to elucidate if the same model could account for the anomalous increase of the orbital eccentricity of the Moon discovered by Williams et al. [14,16]. The Moon also moves along its orbit in the same direction as the rotation of the Earth. Consequently, the momentum of the anomalous forces over the volume of the Earth would be opposite to the Earth's rotational angular momentum and from Eqs. (19), (14) and (18) we find:

$$
\boldsymbol{M}_{\text {Earth }}=-1.46197 \times 10^{23} \beta(D) \text { Joules } .
$$

This corresponds to a decrease of the rotational angular momentum of the Earth or an increase of the Moon's orbital angular momentum of the same magnitude by the principle of angular momentum conservation. Similarly, the associated decrease in the rotational energy of the Earth is given by:

$$
\frac{d E_{\text {rot }}}{d t}=\Omega_{\text {Earth }} M_{\text {Earth }}=-1.0661 \times 10^{19} \beta(D) \text { Joules } / \mathrm{s},
$$

where $M_{\text {Earth }}$ is, as before, the total momentum of the non-Newtonian forces acting upon the Earth. Of course, the Moon's orbital energy changes by a quantity of the same magnitude, but opposite in sign, as a consequence of energy conservation. We have also that the orbital angular momentum of the Moon and its orbital energy are given by:

$$
\begin{aligned}
& H_{\text {Moon }}=m_{\text {Moon }}{ }^{v} \text { Moon } \\
& D_{\text {Moon }}=2.83788 \times 10^{34} \text { Joules } \times \mathrm{s}, \\
& E_{\text {Moon }}=-\frac{\mu_{\text {Earth }} m_{\text {Moon }}}{2 D_{\text {Moon }}}=-3.87317 \times 10^{28} \text { Joules } .
\end{aligned}
$$

It is known from perturbation theory that the variation of the orbital eccentricity, $\epsilon$, as a consequence of perturbing forces can be calculated from the expression [40]:

$$
\frac{d \epsilon}{d t}=-\frac{1-\epsilon^{2}}{2 \epsilon}\left\{\frac{d H}{d t} \frac{2}{H}+\frac{d E}{d t} \frac{1}{E}\right\}
$$

where $E$ is the orbital energy and $H$ is the orbital angular momentum. From Eqs. (23), (24), (25) and (26) and the value $\epsilon=0.0549$ for the eccentricity of the Moon's orbit [39], we can estimate the variation in the eccentricy of the orbit of the Moon as a consequence of the Whitehead's extra non-Newtonian force as follows:

$$
\frac{d \epsilon}{d t}=\beta(D) 2.40571 \times 10^{-9} \mathrm{~s}^{-1},
$$

where we have taken into account that the orbital angular momentum rate for the Moon is opposite to the one given for the Earth in Eq. (23) and that the sign is also opposite to that of Eq. (24) for the orbital energy change. In their last work, Williams et al. [16] gives the value of $\Delta \epsilon / \Delta t=5 \times 10^{-12}$ per year for the anomalous rate of change of the orbital eccentricity, i. e., $\Delta \epsilon / \Delta t=1.58444 \times 10^{-19}$ per second. To be compatible with the result in Eq. (27) we must have $\beta(D)=6.58616 \times 10^{-11}$. We see that this value of the nondimensional parameter $\beta$ is similar to that found in the calculation of the anomalous increase of the astronomical unit in the previous section. 
4.2. The meaning of the parameter $\beta(D)$ Two values of $\beta(D)$ have been found, respectively, in Secs. 4 and 4.1 where we have applied the force model to the increase of the astronomical unit and the anomalous increase of the orbital eccentricity for the Moon's orbit. These values are very similar and in the range $5 \times 10^{-11}<\beta(D)<7 \times 10^{-11}$. A question that arises concerns the meaning of this small parameter. One possibility is that $\beta$ is really a universal constant but it is also possible that the similarity among the two values is, purely, coincidental and that it depends on some orbital parameters such as the distance from the central body.

This second possibility is more interesting if we take into account that the extended Whitehead's theory discussed in this paper predicts values for anomalous energy changes in Earth's flybys in agreement with the observations of Anderson et al. [43]. This was analyzed in previous papers about this theory [41,42]. For this reason, we expect that $\beta(D=R) \simeq 1$ were $R$ is the radius of the central body (the Earth in the case of the flybys) because during a flyby the spacecraft comes very close to the surface of the planet. In other numerical and analytical studies on the flyby anomaly, it has been suggested that the interaction responsible for these anomalies should diminish exponentially with altitude over the surface of the Earth [44]. A fact that is supported by a statistical analysis by Jouannic et al. [45] which showed that the largest values for the flyby anomaly are largely correlated with the time the spacecraft stays below an altitude of $2000 \mathrm{~km}$.

Therefore, we propose a relation of the form:

$$
\beta(D)=A e^{-D / \Lambda},
$$

where $D$ is the distance from the central or source body to the orbiting planet, satellite or spacecraft, and $\Lambda$ is a characteristic length scale that depends of the physical parameters of the source. We can assume that $\Lambda$ is proportional to the radius of the central body, $\Lambda \propto R$. For the case of the Earth, and following previous work on the flyby anomaly $[44,45]$ it seems that the characteristic length for this anomalous interaction is not much larger than the radius of the Earth. For example, if we take $\Lambda_{\text {Earth }}=2.5 R_{\text {Earth }}$ we find from Eq. (28) and the value of $\beta$ found in Sec. 4.1 that $A \simeq 1.3352$.

By using this value of the coefficient $A$ and applying Eq. (28) to the Sun-Earth system we find from the value of $\beta$ given in Sec. 4 that $\Lambda_{\text {Sun }}=8.96482 R_{\text {Sun. }}$. This value is larger in terms of the radius of the central body that the one given for Earth. The same is found if we make another hypothesis about the range of the anomalous interaction for the case of the Earth. A formula for $\Lambda$ would be purely speculative but one can check that:

$$
\Lambda=\frac{5}{2} \frac{\rho_{\text {Earth }}}{\rho} R,
$$

predicts $\Lambda \simeq 9.79 R_{\text {Sun }}$ which suggest that not only the mass and radius of the central body may be important for the extended field but also the density. For the minimal altitude of the NEAR flyby of the Earth, reached on January 23rd, 1998, at $h=532 \mathrm{~km}$ the Eq. (28) yields $\beta \simeq 0.5$, a value of order unity. It has already been shown that for $\beta=1$ the force model in Eq. (7) yields predictions of the correct sign and order of magnitude for the anomalous energy changes in several spacecraft flybys of the Earth $[41,42]$. Consequently, with the exponential cut-off on the range of the interaction we have shown that this model may account for three anomalies in the Solar system: the flyby anomaly, the anomalous increase of the eccentricity of the Moon's orbit and the anomalous increase of the astronomical unit. 


\section{Conclusions}

In a recent review by Iorio [2] a survey of many possible anomalous effects in the Solar system are discussed. These effects have been accumulating thanks to the new avenues of discovery allowed by modern measurement techniques, including radar ranging, laser ranging [46], accurate definition of the ICRS frame using extragalactic sources such as quasars [47], etc. Some of the anomalies could be finally eliminated through higher precision measurements in the near future, which could reduce the statistical errors making then non-significant. Indeed, this is the opinion of some authors [16]. On the other hand, others may be proven significant but explainable within the context of standard physics. The shift in the Doppler effect of the signals received from the antenna of the Pioneer $10 \& 11$ remained unexplained for years but, finally, a recovery of the tapes containing information about the whole trajectory and the accurate modelling of thermal effects provided the generally accepted explanation in terms of anisotropic emission of radiation [48-50].

Notwithstanding this success, other anomalies are still lingering. Among them, we have the anomalous increase of the astronomical unit that appears after processing a large set of ranging data for planets and spacecraft $[3,4]$. This is in the range of a fraction of centimeter per year but still significant at $2 \sigma$, the anomalous increase of the eccentricity of the orbit of the Moon that remains after considering all the detailed modelling of tidal processes in the Earth and the Moon [14,16], is in the range of a few parts of $10^{-12}$ per year and it is still significant at the same level of $2 \sigma$ even after improved modelling $[2,16]$. Finally we have the flyby anomalous, i. e., the anomalous orbital energy changes (positive or negative) that arise in an Earth-centered frame as found after analyzing the Doppler residuals for the ranging and tracking data of the pre-encounter and post-encounter trajectories [43]. These velocity changes are in the range of a few $\mathrm{mm} / \mathrm{s}$ and, for the time being, have not been successfully and convincingly explained.

The fact that there are some anomalies in the behaviour of gravity is not restricted to the Solar system. The anomalous rotation curves of the galaxies have been invoked as a convincing proof of the existence of a new kind of matter that not couples to electromagnetic radiation and, consequently, is called dark matter. Anyway, many authors propose that a modified theory of gravity may account for these anomalies without invoking any kind of dark matter [51-55]. For all these reasons, it is not far-fetched to believe that General Relativity could be an incomplete theory, and not only in the sense that it remains inconsistent with quantum theory, but even from a purely classical point of view.

In this paper we have shown that an extended Whitehead model with a truncation exponential term may account for three anomalies in the Solar system: (i) the astronomical unit anomaly (ii) the anomalous increase of the eccentricity of the orbit of the Moon (iii) the flyby anomaly. Encompassing all these effects within a single model suggests that a precise modified theory of gravity is behind them. To achieve this ambitious objective it would be necessary, in the first place, an improvement of the accuracy of the measurements of these possibly anomalous effects. A satellite with a highly elliptical orbit with a low perigee could allow for a systematic analysis of flyby anomalies in its pass through the perigee [56]. Obtaining more data with improved lunar laser ranging techniques will allow for reducing the statistical error in the measurement of the evolution of the Moon's orbital eccentricity and the enhancement of tidal models is likely to establish in a near future the reality of the anomalous increase of the eccentricity or its reduction below the level of noise [16]. 
In any case, the model discussed in this paper could allow to obtain other independent predictions that could be verified and give additional support to the effects of modified gravity in the scale of the Solar system. Moreover, an extended theory, consistent with the principles of general relativity, that could explain all these anomalies beyond our current understanding of gravity is still to be found.

\section{Acknowledgements}

The author gratefully acknowledges Ll. Bel for many useful discussions on the topic of the flyby anomaly and for developing the extended Whitehead's theory discussed in this paper.

\section{Conflicts of Interest}

The author declare no conflict of interest.

\section{References}

1. Will, C. M. The Confrontation between General Relativity and Experiment. Living Rev. Relativity 2006, 9,3 .

2. Iorio, L. Gravitational anomalies in the Solar System ?. International Journal of Modern Physics D 2015, 24, 1530015. arXiv: 1412.7673

3. Krasinsky, G.A.; Brumberg, V.A. Secular increase of astronomical unit from analysis of the major planet motions, and its interpretation. Celestial Mechanics \& Dynamical Astronomy, 2004, 90, 267-288.

4. Standish, E. M. The astronomical unit now, in Kurtz, D.W. (Ed.), Transit of Venus: New Views of the Solar System and Galaxy, Proceedings IAU Colloquium; Cambridge University Press, Cambridge, p. 163.

5. Resolution B2 on the redefinition of the astronomical unit of length, International Astronomical Union. Available online at: https://www.iau.org/static/resolutions/IAU2012_English.pdf

6. Iorio, L. Secular increase of the astronomical unit and perihelion precessions as tests of the dvali-gabadadze-porrati multi-dimensional braneworld scenario. Journal of Cosmology and Astroparticle Physics 2005, 09. arXiv:gr-qc/0508047

7. Li, X.; Chang, Z. Kinematics in randers-finsler geometry and secular increase of the astronomical unit. Chinese Physics C 2011, 35, 914. arXiv:0911.1890

8. Arakida, H. Application of time transfer function to mcvittie spacetime: Gravitational time delay and secular increase in astronomical unit. General Relativity \& Gravitation 2011, 43, 2127-2139. arXiv:1103.2569

9. Miura, T.; Arakida, H.; Kasai, M.; Kuramata, S. Secular increase of the astronomical unit: a possible explanation in terms of the total angular momentum conservation law. Publ. Astron. Soc. of Japan 2009, 61, 1247. arXiv:0905.3008.

10. Stephenson, F. R. Tidal recession of the Moon from ancient and modern data. Journal of the Bristish Astronomical Association 1981, 91, 136-147.

11. Acedo, L. A phenomenological variable speed of light theory and the secular increase of the astronomical unit. Phys. Essays 2013, 26, 567-573. 
12. Bel, Ll. Earth and Moon orbital anomalies, arXiv:gr-qc/1402.0788, 2014.

13. Acedo, L. Anomalous post-Newtonian terms and the secular increase of the astronomical unit. $A d v$. Space Res. 2013, 52, 1297-1303. arXiv:1401.4056.

14. Williams, J.G.; Boggs, D.H. Lunar Core and Mantle. What Does LLR See? In Proceedings of the 16th International Workshop on Laser Ranging, Poznan, Poland, 13-17 October 2008.

15. Williams, J. G.; Boggs D. H.; Folkner W. M. DE430 Lunar Orbit, Physical Librations, and Surface Coordinates Jet Propulsion Laboratory IOM 335-JW,DB,WF-20080314-001 2013. Available online at: http://proba2.sidc.be/aux/data/spice/docs/DE430_Lunar_Ephemeris_and_Orientation.pdf. (Accessed on June, 12th, 2015).

16. Williams,J. G.; Turyshev, S. G.; and Boggs, D. H. The past and present Earth-Moon system: the speed of light stays steady as tides evolve. Planetary Science 2014, 3, 2.

17. Iorio, L. On the anomalous secular increase of the eccentricity of the orbit of the moon. Mon. Not. Roy. Astron. Soc. 2011, 415, 1266-1275. arXiv:1102.0212.

18. Iorio, L. An empirical explanation of the anomalous increases in the astronomical unit and the lunar eccentricity. Astron. J. 2011, 142, 68. arXiv:1102.4572.

19. Acedo, L. Anomalous post-newtonian terms and the secular increase of the astronomical unit. Adv. Space Research 2013, 7(1), 1297-1303. arXiv: 1401.4056

20. Anderson, J. D.; Campbell, J. K.; Ekelund, J. E.; Ellis, J.; Jordan, J. F. Anomalous orbital-energy changes observed during spacecraft flybys of earth. Phys. Rev. Lett. 2008, 100(9), 091102.

21. Adler, S. L. Can the flyby anomaly be attributed to earth-bound dark matter?. Phys. Rev. D 2009, 79, 023505. arXiv:0805.2895v4

22. Pinheiro, M. J. The flyby anomaly and the effect of a topological torsion current. Phys. Lett. A 2014, 378, 3007-3011. arXiv:1404.1101

23. Acedo, L. The flyby anomaly: a case for strong gravitomagnetism ?. Adv. Space Res. 2014, 54, 788-796. arXiv: 1505.06884

24. Lämmerzahl, C.; Preuss, O.; Dittus, H. Is the physics of the Solar System really understood ?. Lasers, Clocks and Drag-Free Control. Astrophysics and Space Science Library 2008, 349, 75-101. arXiv:gr-qc/0604052

25. Iorio, L. A flyby anomaly for Juno ?. Not from standard physics. Adv. Space Res. 2014, 54(11), 2441-45. arXiv: 1311.4218

26. Iorio, L. The effect of General Relativity on Hyperbolic Orbits and Its Application to the Flyby Anomaly. Scholarly Research Exchange 2009, ID807695, 1-8.

27. Cartwright, D. E. Tides: A scientific history. Cambridge University Press, Cambridge, UK, 2001.

28. Acedo, L. On the effect of ocean tides and tesseral harmonics on spacecraft flybys of the Earth. Mon. Not. Roy. Astron. Soc. 2016, 2(1), 2119-2124. arXiv: 1611.08142

29. Penrose, R.; Floyd, R. M. Extraction of rotational energy from a Black Hole. Natural Physical Science 1971, 229, 177.

30. Nordström, G. Zur Theorie der Gravitation vom Standpunkt des Relativitätsprinzips. Annalen der Physik 1913, 347(13), 533-554. 
31. Norton, J. D. Einstein, Nordström and the early demise of scalar, Lorentz covariant theories of gravitation, in Theories of Gravitation in the Twilight of Classical Physics. Part I. The Genesis of General Relativity Vol 3, Renn, J. (Ed.), Kluwer Academic Publishers, The Netherlands, 2005.

32. Whitehead, A. N. The Principle of Relativity; Cosimo, Inc., New York, 2007

33. Coleman, A. J. Whitehead's principle of Relativity, arXiv:physics/0505027v2, 2005.

34. Bain, J. Whitehead's Theory of Gravity. Stud. Hist. Phil. Mod. Phys. 1998, 29(4), 547-574.

35. Reinhardt, M.; Rosenblum, A. Whitehead contra Einstein. Phys. Lett. A 1974, 48(2), 115-116.

36. Gibbons, G.; Will, C. M. On the multiple deaths of Whitehead's Theory of Gravity. Stud. Hist. Phil. Mod. Phys. 2008, 39(1), 41-61. arXiv:gr-qc/0611006v1

37. Bel, Ll. A look inside the theory of the linear approximation, arXiv:gr-qc/0605057v3, 2007.

38. Bel, Ll. A new look inside the theory of the linear approximation: Gravity assists and Flybys, unpublished paper. Available online at http://www.lluisbel.com/upload/OnHold/FlyBys.pdf, 2015.

39. Williams, D. R. Planetary Fact Sheet, https://nssdc.gsfc.nasa.gov/planetary/factsheet/ (Accessed September 9th, 2018)

40. Burns, J. A. Elementary derivation of the perturbation equations of celestial mechanics. American Journal of Physics 1976, 44, 944-949

41. Acedo, L. The flyby anomaly in an extended Whitehead's theory. Galaxies 2015, 3(3), 113-128.

42. Acedo, L.; Bel, Ll. On a correlation among azimuthal velocities and the flyby anomaly sign. Astronomische Nachrichten 2017, 338(1), 117-124. arXiv: 1602.03669

43. Anderson, J. D.; Campbell, J. K.; Ekelund, J. E.; Ellis, J.; Jordan, J. F. Anomalous Orbital-Energy Changes Observed during Spacecraft Flybys of Earth. Phys. Rev. Lett. 2008, 100, 091102.

44. Acedo, L. Anomalous accelerations in spacecraft flybys of the Earth. Astrophysics and Space Science 2017, 362(12), 1-15. arXiv: 1711.02875

45. Jouannic, B.; Noomen, R.; van den IJSel; J. A. A. The flyby anomaly: An investigation into potential causes. In Proceedings of the 25th International Symposium on Space Flight Dynamics ISSFD, Munich (Germany), 2015.

46. Dickey, J. O.; Bender, P. L.; Faller, J. E.; Newhall, X. X.; Ricklefs, R. L.; Ries, J. G.; Shelus, P. J.; Veillet, C.; Whipple, A. L.; Wiant, J. R.; Williams, J. G.; Yoder, C. F. Lunar Laser Ranging: A Continuing Legacy of the Apollo Program. Science 1994, 265, 482-490.

47. Gaia Collaboration; Brown A. G. A. et al. Gaia Data Release 2: Summary of the contents and survey properties. Astronomy \& Astrophysics 2018, 616(A1). arXiv: 1804.09365

48. Bertolami, O.; Francisco, F.; Gil, P. J. S.; Páramos, J. Estimating Radiative Momentum Transfer Through a Thermal Analysis of the Pioneer Anomaly. Space Science Reviews 2010, 151, 75-91. arXiv: 0809.2633

49. Rievers, B.; Lämmerzahl, C. High precision thermal modeling of complex systems with application to the flyby and Pioneer anomaly. Annalen der Physik 2011, 523, 439-449. arXiv: 1104.3985

50. Turyshev, S. G.; Toth, V. T.; Kinsella, G.; Lee, S. C.; Lok, S. M.; Ellis, J. Support for the Thermal Origin of the Pioneer Anomaly. Physical Review Letters 2012, 108(24), 241101. arXiv: 1204.2507

51. Moffat, J. W.; Rahvar, S.; Toth, V. T. Applyling MOG to lensing: Einstein rings, Abell 250 and the Bullet Cluster. Galaxies 2018, 6(2), 43. arXiv: 1204.2985 
52. Wojnar, A.; Sporea, C. A.; Borowiec, A. A simple model for explaining galaxy rotation curves. Galaxies 2018, 6(3), 70. arXiv: 1804.09620

53. Acedo, L. Modified Newtonian Gravity as an alternative to the Dark Matter hypothesis. Galaxies 2017, 5(4), 74.

54. Kroupa, P. Galaxies as simple dynamical systems: observational data disfavor dark matter and stochastic star formation. Canadian Journal of Physics 2015, 93(2), 169-202. arXiv: 1406.4860

55. Sanders, R. H.; McGaugh, S. S. Modified Newtonian dynamics as an alternative to Dark matter. Ann. Rev. Astron. Astrophys. 2002, 40, 263. arXiv: astro-ph/0204521v1

56. Páramos, J.; Hechenblaikner, G. Probing the Flyby Anomaly with the future STE-QUEST mission. Planetary and Space Science 2013, 79-80. arXiv: 1210.7333 